\title{
Protocol
}

\section{Tracking Movement Behavior of Multiple Worms on Food}

\author{
Eviatar Yemini, Rex A. Kerr, and William R. Schafer
}

MATERIALS

Neurobiological research in genetically tractable organisms relies heavily on robust assays for behavioral phenotypes. The simple body plan of the nematode Caenorhabditis elegans makes it particularly amenable to the use of automated microscopy and image analysis to describe behavioral patterns quantitatively. Forward genetic screens and screens of drug libraries require high-throughput phenotyping, a task traditionally incompatible with manual scoring of quantitatively varying behaviors. Highthroughput automated analysis of C. elegans movement behavior is now possible with several different tracking software packages. The Multiworm Tracker (MWT) described here is designed for highthroughput analysis: it can record dozens of worms simultaneously at 30 frames per second for hours or days at a time. This is accomplished by performing all image analysis in real time, saving only the worm centroid, bearing, and outline data to the disk. To simplify image processing, the system focuses only on worms that have moved, and detects and discards worms that are touching rather than trying to isolate them computationally. Because the software is entirely automated, protocols can run unattended once the worms have been placed and the software has been started. The MWT does not save images for later analysis, but behavior can be validated manually with a companion analysis tool that replays recorded body postures. This protocol describes a basic basal movement assay on food using the MWT; similar protocols apply to related assays and to similar multiple animal trackers. The protocol can be extended to a variety of assays ranging from tap response to chemotaxis.

It is essential that you consult the appropriate Material Safety Data Sheets and your institution's Environmental Health and Safety Office for proper handling of equipment and hazardous materials used in this protocol.

RECIPE: Please see the end of this article for recipes indicated by $<\mathrm{R}>$. Additional recipes can be found online at http://cshprotocols.cshlp.org/site/recipes.

Reagents

C. elegans, Bristol N2, adult hermaphrodites

Nematode growth medium $(\mathrm{NGM})<\mathrm{R}>$

For liquid medium, omit the agar.

OP50 Escherichia coli

Equipment

Computer with at least 2 GB RAM and $1680 \times 1050$ or $1600 \times 1200$ monitor

Dalsa Falcon 4 M30 camera $(2352 \times 1728$ at $31 \mathrm{~Hz})$ with M42 to F-Mount lens adapter

Adapted from Imaging in Neuroscience (ed. Helmchen and Konnerth). CSHL Press, Cold Spring Harbor, NY, USA, 2011.

(C) 2011 Cold Spring Harbor Laboratory Press

Cite this article as Cold Spring Harbor Protoc; 2011; doi:10.1101/pdb.prot067025 
E. Yemini et al.

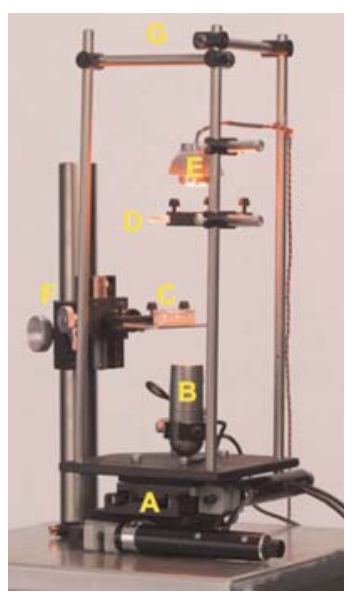

FIGURE 1. Hardware: (A) The stage, composed of $x$ - and $y$-axis actuators and linear translation stages; $(B)$ the camera; $(C)$ the sample, a worm on an agar Petri dish; $(D)$ a diffuser above a collimating Fresnel lens, to provide uniform illumination; $(E)$ a highintensity red light-emitting diode (LED) for illumination; $(F)$ coarse and fine focusing knobs to focus the sample in view of the camera; $(G)$ a rigid cage to center the illumination above the camera and eliminate vibrations caused by moving the stage.

Multiworm Tracker software (http://sourceforge.net/projects/mwt)

National Instruments NI PCIe-1427 image capture card

Petri dishes, $\geq 5 \mathrm{~cm}$

Plate holder, $\sim 15 \mathrm{~cm}$ above light, $\sim 30 \mathrm{~cm}$ below camera

Rodenstock $60 \mathrm{~mm}, \mathrm{f} / 4.0$ Rodagon lens with MFB F-mount adapter, modular focus block ( $25 \mathrm{~mm})$, M39 $\times 0.75$ lens adapter

Schott ACE light source with fiber-optic backlight $(4 \times 4.88$ in. $)$

The complete hardware setup, including stage, camera, and light source, is shown in Figure 1.

1. Set up the recording system, the Multiworm Tracker (MWT), as described in its user manual, and use the Measurement and Automation Explorer tool to set the camera to 8 bit resolution.

2. Measure the size of a pixel in millimeters: View a ruler with the camera and compute, for example [number of pixels in $1 \mathrm{in.}] / 25.4$.

3. Prepare worms for tracking.

i. Seed one plate of NGM with $50 \mu \mathrm{L}$ of NGM media, spread to cover the center of the plate.

ii. Leave plate overnight.

iii. Select five young adult hermaphrodites and transfer them onto the NGM plate. Incubate them for $3 \mathrm{~h}$ to lay eggs, and then remove the adult hermaphrodites.

iv. Allow the eggs to grow to young adults (72-96 h).

4. Place the plate in position for imaging with the lid on, making sure that the worms are in focus with the camera, that the brightness across the field of view varies by no more than $\sim 40 \%$, and that the brightest pixels are below saturation (a good target is 200-220 out of 255).

See Troubleshooting.

5. Run the tracker.

i. In the MWT software, select a target directory, an experiment title, and a duration to record.

ii. Verify in the image window that the MWT is detecting the worms.

See Troubleshooting.

iii. Press "Go," and wait for capture to complete. 
6. Analyze the resulting data with the companion command-line Choreography program as described in the MWT user manual.

i. Supply the pixel size measured in Step 2 with the -p option (e.g., -p 0.024 for a setup with $24 \mu \mathrm{m} /$ pixel, which is approximately what it should be with the imaging setup described).

ii. Request the output parameters desired (e.g., -o Ns will produce a table containing the number of worms and the mean speed of those worms at each time point).

See the users guide for more detail.

\section{TROUBLESHOOTING}

Problem (Steps 4 and 5.ii): The worms are obscured by condensation on the lid of the plate.

Solution: Consider the following:

1. Check the temperature in the room; if the lid of the plate is colder than the base, condensation will appear. The light source box may need to be moved farther away or downwind instead of upwind; the light plate itself should heat the base enough to cause fogging.

2. Remove the lid from the Petri dish. Be aware, however, that worms are highly sensitive to drying, and long assays $(>1 \mathrm{~h})$ will be measuring predominantly a drying-related response unless the humidity is very high.

Problem (Step 5.ii): The MWT fails to show any image of worms.

Solution: Consider the following:

1. Check that the Measurement and Automation Explorer tool can find the camera and view images from it; then close the Explorer and try the MWT again.

2. The National Instrument Vision Runtime License has not been activated and the trial period has expired. Contact National Instruments to purchase a runtime license.

Problem (Step 5.ii): The tracker cannot find the worms.

Solution: Consider the following:

1. Make sure there is good contrast between the worm and the background. With the optical setup as described, contrast should be $\sim 50 \%$.

2. Make sure the lighting is bright but does not saturate the camera.

3. Alter the tracking parameters such as intensity threshold within the MWT.

Problem (Step 5.ii): The tracker finds many tiny worms as well as the adult worms. Solution: Consider the following:

1. Increase the minimum object size in the MWT.

2. Raise the worms at a colder temperature or run the experiment before any eggs have hatched.

3. Select and transfer adult worms to the plate instead of waiting for them to grow from eggs. Allow at least $2 \mathrm{~h}$ to elapse between transfer and recording, if possible.

Worm trackers fall into two general types. Single-worm trackers follow individual animals at relatively high magnification, keeping them in the field of view by moving the camera or a motorized stage. Such systems have been used to study aspects of locomotion such as turning and speed (Pierce-Shimomura et al. 1999) as well as body posture and morphology (Karbowski et al. 2008). MWTs track multiple individuals in populations, by necessity at lower magnification, and usually focus on locomotion 

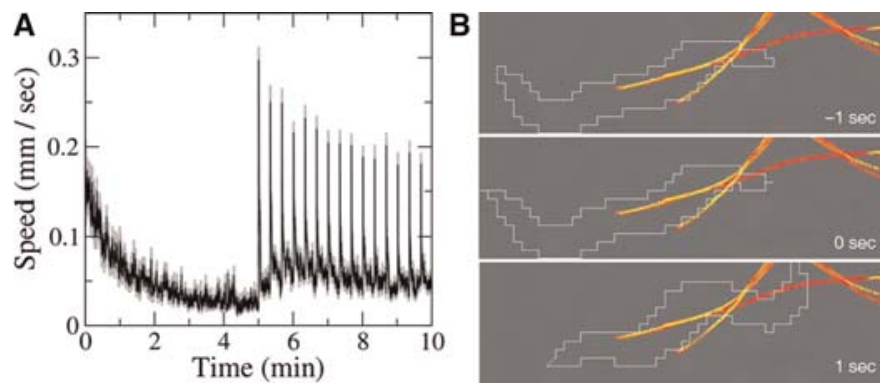

FIGURE 2. A tap habituation assay performed on the MWT. (A) Average speed of $\sim N$ worms over 10 min with plate tap delivered at $5 \mathrm{~min}$ and $3 \times / \mathrm{min}$ thereafter (15 taps total). Black line is mean; standard error in gray. $N=45-50$ animals per time point (one plate). (B) Manual validation of tap response with worm position 1.0 sec before (top), at (center), and $1.0 \mathrm{sec}$ after (bottom) a tap. Colored dots are centroid positions colored by speed, where red is slower and yellow faster; one dot is drawn every $33 \mathrm{msec}$.

features. Generally speaking, single-worm trackers allow one to measure a greater number of parameters, whereas MWTs offer much higher efficiency and throughput. A discussion of the principles of single-worm tracking, along with a protocol, can be found in Preparation of Samples for SingleWorm Tracking (Yemini et al. 2011a). A protocol is also available for Illumination for Worm Tracking and Behavioral Imaging (Yemini et al. 2011b).

This protocol describes the use of the software package MWT, and discusses possible applications for genetic analyses of behavior. Neuronal tracking uses precisely the same algorithm to search for labeled neurons. The few minor changes this protocol may require involve using higher magnification to view tracked neurons and inverting the binary thresholding to discover bright neurons on a dark background (worms are usually imaged in bright-field, where they are darker than their background).

A variety of methods for recording many worms simultaneously have been developed recently. Image analysis methods used on single worms can be applied to high-resolution images of whole plates of worms to give similar data: some individual measurements are noisier or impossible, but collecting large sample sizes is much faster. Ramot et al. (2008) have created the Parallel Worm Tracker, a suite of three related open-source programs that acquire image data, perform basic image processing to identify worms and produce tracks of the worms's centroid positions, and analyze those tracks to compute linear and angular velocity. Similarly, Roussel et al. (2007) and Tsechpenakis et al. (2008) have created software that analyzes stored image data with more sophisticated algorithms that account for noise and adjacent worms and can provide basic postural data in addition to centroid position. Other efforts at simultaneous worm tracking have focused on worms in 96-well plates with no (Simonetta and Golombek 2007) or very minimal (Buckingham and Sattelle 2009) image processing, or on robustly detecting pairs of worms in contact for detailed assays. The MWT discussed here is conceptually very similar to the trackers described by Ramot et al. (2008) and Tsechpenakis et al. (2008), save for its focus on real-time image processing.

Multiworm tracking is possible with a wide variety of cameras and imaging speeds. For the MWT, the primary two requirements are that worms must overlap over at least $50 \%$ of their bodies from one frame to the next, and must be large enough to cause a clear change in brightness when moving into a new area of the plate. Thus, frame rates slower than $\sim 0.5 \mathrm{~Hz}$ for crawling or $\sim 10 \mathrm{~Hz}$ for swimming (because the worm swings side to side) will tend to fail because of excessive movements; magnifications that result in pixels that span more than $\sim 60 \mu \mathrm{m}$ also tend to give substandard results.

TABLE 1. Choreography analysis parameters used in the example results shown in Figure 2

\begin{tabular}{lllll}
\hline Pixel size & $\begin{array}{l}\text { Speed averaging } \\
\text { window }\end{array}$ & $\begin{array}{l}\text { Object min } \\
\text { duration }\end{array}$ & $\begin{array}{l}\text { Object min } \\
\text { movement }\end{array}$ & Output quantities \\
\hline $0.0243 \mathrm{~mm} / \mathrm{sec}$ & $0.2 \mathrm{sec}$ & $20 \mathrm{sec}$ & 2 body lengths & $\begin{array}{l}\text { ss*s (speed: mean, standard deviation, } \\
\text { number of samples) }\end{array}$ \\
\hline
\end{tabular}


Converting a single-worm manual assay to an automated assay can be a considerable amount of work. The MWT does not automatically detect qualitative phenotypes often scored manually (e.g., "kinker"), so a quantitative proxy must be discovered. For example, for tap habituation assays, reversal distance is a typical metric (Broster and Rankin 1994), but is relatively difficult to extract computationally. However, for longer interstimulus tap intervals, increase in speed of animals on food is a good alternative metric for response to tap (Fig. 2A; the transient increase in speed due to manipulation of the plate before the beginning of the experiment is typical). When beginning a new assay, it is valuable to compare manually scored behaviors with automated results. To assist in this, the Choreography program contains a -map option that brings up a map browser interface, which allows one to replay the movements of the tracked worms (Fig. 2B). The analysis parameters and exclusion criteria used in the results shown in Figure 2 are listed in Table 1.

RECIPE

Nematode Growth Medium (NGM)

\begin{tabular}{lll} 
Reagent & $\begin{array}{l}\text { Amount to } \\
\text { add (for 1 L) }\end{array}$ & $\begin{array}{l}\text { Final } \\
\text { concentration }\end{array}$ \\
\hline Agar & $17.0 \mathrm{~g}$ & $1.7 \%(\mathrm{w} / \mathrm{v})$ \\
$\mathrm{NaCl}$ & $2.9 \mathrm{~g}$ & $50 \mathrm{~mm}$ \\
Peptone & $2.5 \mathrm{~g}$ & $0.25 \%(\mathrm{w} / \mathrm{v})$ \\
$\mathrm{CaCl}_{2}(1 \mathrm{M})$ & $1 \mathrm{~mL}$ & $1 \mathrm{~mm}$ \\
$\mathrm{Cholesterol}(5 \mathrm{mg} / \mathrm{mL})$ & $1 \mathrm{~mL}$ & $5 \mu \mathrm{mL}$ \\
$\mathrm{KH}_{2} \mathrm{PO}_{4}(1 \mathrm{M})$ & $25 \mathrm{~mL}$ & $25 \mathrm{~mm}$ \\
$\mathrm{MgSO}_{4}(1 \mathrm{M})$ & $1 \mathrm{~mL}$ & $1 \mathrm{~mm}$
\end{tabular}

Mix the first three reagents and autoclave. After the mixture is cool, add the last four reagents.

\section{REFERENCES}

Broster BS, Rankin CH. 1994. Effects of changing interstimulus interval during habituation in Caenorhabditis elegans. Behav Neurosci 108: 1019-1029.

Buckingham SD, Sattelle DB. 2009. Fast, automated measurement of nematode swimming (thrashing) without morphometry. BMC Neurosci 10: 84 .

Karbowski J, Schindelman G, Cronin CJ, Seah A, Sternberg PW. 2008. Systems level circuit model of C. elegans undulatory locomotion: Mathematical modeling and molecular genetics. J Comput Neurosci 24: 253-276.

Pierce-Shimomura JT, Morse TM, Lockery SR. 1999. The fundamental role of pirouettes in Caenorhabditis elegans chemotaxis. J Neurosci 19: 9557-9569.

Ramot D, Johnson BE, Berry TL Jr, Carnell L, Goodman MB. 2008. The Parallel Worm Tracker: A platform for measuring average speed and drug-induced paralysis in nematodes. PLoS One 3: e2208.
Roussel N, Morton CA, Finger FP, Roysam B. 2007. A computational model for C. elegans locomotory behavior: Application to multiworm tracking. IEEE Trans Biomed Eng 54: 1786-1797.

Simonetta SH, Golombek DA. 2007. An automated tracking system for Caenorhabditis elegans locomotor behavior and circadian studies application. J Neurosci Methods 161: 273-280.

Tsechpenakis G, Bianchi L, Metaxas D, Driscoll M. 2008. A novel computational approach for simultaneous tracking and feature extraction of C. elegans populations in fluid environments. IEEE Trans Biomed Eng 55: $1539-1549$.

Yemini E, Kerr RA, Schafer WR. 2011a. Preparation of samples for singleworm tracking. Cold Spring Harb Protoc doi: 10.1101/pdb.prot066993.

Yemini E, Kerr RA, Schafer WR. 2011b. Illumination for worm tracking and behavioral imaging. Cold Spring Harb Protoc doi: 10.1101/pdb. prot067009. 


\section{Tracking Movement Behavior of Multiple Worms on Food}

Eviatar Yemini, Rex A. Kerr and William R. Schafer

Cold Spring Harb Protoc; doi: 10.1101/pdb.prot067025

\begin{tabular}{|c|c|}
\hline $\begin{array}{l}\text { Email Alerting } \\
\text { Service }\end{array}$ & Receive free email alerts when new articles cite this article - click here. \\
\hline $\begin{array}{l}\text { Subject } \\
\text { Categories }\end{array}$ & $\begin{array}{l}\text { Browse articles on similar topics from Cold Spring Harbor Protocols. } \\
\text { Behavioral Assays ( } 99 \text { articles) } \\
\text { C. elegans (45 articles) } \\
\text { Image Analysis ( } 124 \text { articles) } \\
\text { Imaging for Neuroscience (342 articles) } \\
\text { Imaging/Microscopy, general (579 articles) } \\
\text { In Vivo Imaging (334 articles) } \\
\text { Video Imaging / Time Lapse Imaging (171 articles) }\end{array}$ \\
\hline
\end{tabular}

